\title{
Design of an Experimental Platform for an X-by-wire Car with Four-wheel Steering
}

\author{
Martin Eder and Alois Knoll
}

\begin{abstract}
In times of increasing fuel price and environmental consciousness coming to the fore, automotive industry is forced to review its existing concepts of building cars based on combustion engines. In addition to other technologies like hydrogen or fuel cell technologies, electric cars with highly efficient battery power supply play a fundamental role. Especially electric cars with wheel hub drive offer great flexibility of car design and approve completely new mobility concepts.

Our contribution to this trend is the setup of a new electric $\mathrm{X}$-by-wire car, characterized by four synchronously controlled wheel drives and four steering drives, which is meant to be a development environment for both mechanics respectively electronics and software. As this prototypic construction allows a full vertical rotation of each wheel, new movement abilities result with demanding steering tasks, as far as control itself and safeguarding against failure is concerned. For instance, the car cannot just direct into a curve, the car can also drive sideways or rotate around arbitrary pivot points.

This paper presents a prototypic implementation of such an electric car, ready to serve as a basement for innovative mobility concepts for the future.
\end{abstract}

Index Terms-Electric vehicle, in-wheel drive, X-by-wire, prototype, four-wheel steering.

\section{INTRODUCTION}

In the past years, big efforts were made to develop cars with less power consumption. Especially alternative technological concepts like hydrogen vehicles, hybrid cars or fully electric vehicles with fuel cells or batteries are discussed more and more [1], [2]. Furthermore, life-cycle costs and environmental effects are considered [3]. New inventions, primarily in battery technology by companies like $A 123$ Systems [4], as well as new mobility concepts of companies such as better place [5], redefine car concepts. There seems to be a change in the appreciation of what cars have to look like and how mobility concepts will work with respect to ecological and economic aspects. Lee and Wong [6] describe such scenarios affecting a wider use of electrical vehicles, pointing out the importance of creativity and strategic design to also adapt to society's needs.

Undeniable is that electric engines are more efficient than combustion engines. Companies like Tesla Motors Inc. or Mitsubishi Motors Corp. either already sell such cars or are at least close to mass-production, implementing latest inventions of lightweight battery systems with high energy density.

Electrically driven cars offer various opportunities for new concepts. Especially electric vehicles with wheel hub drive

M. Eder and A. Knoll are with the Department of Robotics and Embedded Systems, Institut für Informatik, Technische Universität München, 85748 Garching, Germany. Corresponding author: ederma@in.tum.de units have the advantage of great flexibility of installation space, for there are no more mechanical connections necessary between particular devices, e.g. within the drive train. Hence, this field of technology has great prospect.

Within the scope of this paper, section II briefly describes some relevant state of the art vehicles, followed by detailed illustration of the mechanical design of our car in section III. Furthermore, in section IV a hardware and software outline is presented, whereas section $\mathrm{V}$ shows the results of our approach. In a final step, conclusions and future works that still remain to be done in our electric vehicle project are summarized in section VI.

\section{STATE OF THE ART}

Meanwhile, there are quite a lot of companies offering or at least announcing innovative electric cars or concepts. As our focus later on will be X-by-wire electric car technology with four-wheel drive including four-wheel steering, exemplarily just a few reference car concepts shall be shortly approached. For more information, refer to [7], [8].

Nissan's Pivo 2 concept car [9] reveils a new car offering four controllable wheels together with a revolving cabin, first announced at the 2007 Tokyo Motor Show. In contrast to our chassis, the wheels can just turn up to $90^{\circ}$. Basic idea is to have a ball-shaped car being able to pivot around the undercarriage. Another interesting car concept occured even in the seventies, when the first series vehicle Honda Prelude with four-wheel steering was built in order to improve cornering ability, but the rotational angle of the rear-wheels was limited to less than $3^{\circ}$. Other automobile manufacturers followed with the integration of electronic control of such wheels, e.g. Renault's Laguna GT. But nevertheless, none of these vehicles have real independend four-wheel drive with steer angles more than $90^{\circ}$.

In what follows, the task to build an electric car prototype based on four-wheel drive with steer angles to the greatest extent unlimited is described, all controlled by-wire.

\section{MECHANICAL DESIGN}

The focus of attention within the mechanical construction is the special development of four drive modules, each being identical. In contrast, the car's chassis is based on a standard unit assembly system with aluminium profiles (AlMgSi), offering the best variation possibilities at low cost. Lightweight electric car design is a crucial issue in order to counterbalance the heavy batteries. Given that the design is adequate, Aluminium and its derivative alloys are very promising in the domain of innovative car-design [10]. 
One drive module consists of a combination of a traction drive and a steering drive. All four modules and the frame of the car establish a very flexible kinematics, being able to fulfill far more movements than conventional cars. Tab. I highlights the most important technical data of the whole construction at a glance.

TABLE I

OVERVIEW OF TECHNICAL DATA OF THE ELECTRIC CAR

\begin{tabular}{|c|c|}
\hline feature & value \\
\hline $\begin{array}{l}\text { number of driven wheels } \\
\text { number of steered wheels }\end{array}$ & $\begin{array}{l}4 \\
4\end{array}$ \\
\hline $\begin{array}{l}\text { payload } \\
\text { weight (including batteries) } \\
\text { mass moment of inertia roll/pitch/yaw axis }{ }^{a}\end{array}$ & $\begin{array}{l}200 \mathrm{~kg} \\
600 \mathrm{~kg} \\
150 / 281 / 286 \mathrm{kgm}^{2}\end{array}$ \\
\hline $\begin{array}{l}\text { length / height / width } \\
\text { center of gravity } b \\
\text { spring travel }\end{array}$ & $\begin{array}{l}2.2 / 1.7 / 1.3 \mathrm{~m} \\
0.6 \mathrm{~m} \\
75 \mathrm{~mm}\end{array}$ \\
\hline $\begin{array}{l}\text { maximum velocity } \\
\text { acceleration time }(0-15 \mathrm{~m} / \mathrm{s}) \\
\text { maximum steer angle } \\
\text { maximum steer angle velocity }\end{array}$ & $\begin{array}{l}15 \mathrm{~m} / \mathrm{s} \\
3.2 \mathrm{~s} \\
360^{\circ} \\
600^{\circ} / \mathrm{s}\end{array}$ \\
\hline $\begin{array}{l}\text { nominal power } \\
\text { maximum drive torque } \\
\text { supply voltage } \\
\text { capacity of batteries }{ }^{c} \\
\text { estimated range }\end{array}$ & $\begin{array}{l}4 \times 1.8 \mathrm{~kW} \\
4 \times 160 \mathrm{Nm} \\
48 \mathrm{~V} \mathrm{DC} \\
140 \mathrm{Ah} \\
50 \mathrm{~km}\end{array}$ \\
\hline
\end{tabular}

${ }^{a}$ relating to center of gravity

${ }^{b}$ distance of center of gravity related to basement

${ }^{c}$ for cost reasons lead acid batteries are used

\section{A. Chassis}

In order to have a perfect platform for future developments, a modular chassis construction with standard aluminium profiles is used, because devices can be easily mounted or changed subsequently.

The embodiment of the chassis is due to the maximum installation space for devices like batteries, steer and drive units, console etc. Our automotive body shell is a combination of simple plastics panels, each of them removeable. For indoor test purpose, additional supporting feet can be moved out to jack the wheels up. Fig. 1 and Fig. 2 give the reader an impression of the chassis construction (frame without plastics panels) and the setup of the whole car.

\section{B. Traction Drive Unit}

The traction drive unit is based on a wheel hub motor, an air-cooled permanent magnet synchronous motor with 36 poles (Perm Motor PRA 230). In addtition to its great drive rating, the motor makes it possible to develop a simple chassis construction, as the motor itself offers a flange for mounting a wheel and a shaft to be fixed to the frame of the car. Because of highly efficient energy transmission without the need of an additional gearbox, this motor has enough power to meet accordant velocity and acceleration demands.

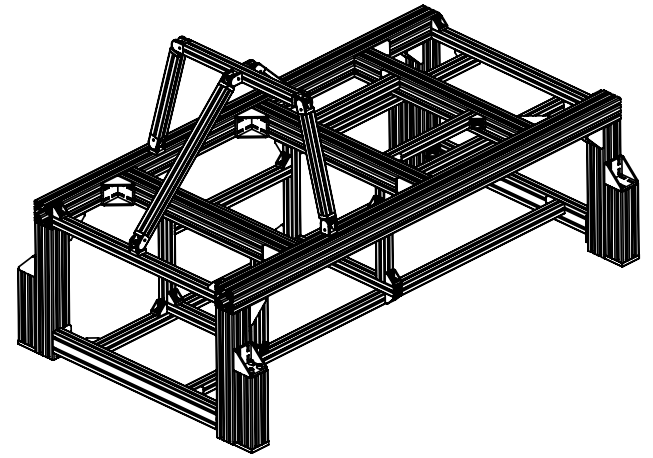

Fig. 1. electric car chassis

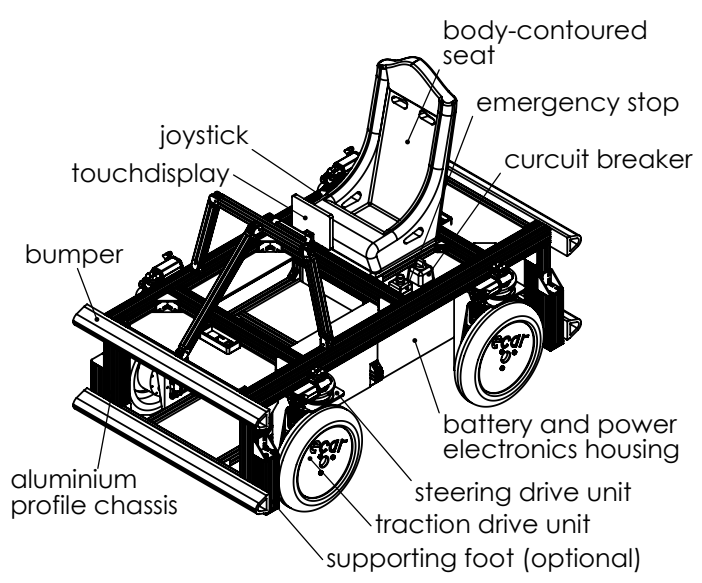

Fig. 2. electric car bodywork

As the motor controllers ACD 4805 get by on $48 \mathrm{~V} \mathrm{DC}$ input voltage out of four automotive batteries (lead acid batteries), each with $12 \mathrm{VDC}$, pretty high currents result to reach a nominal power of $1.8 \mathrm{~kW}$ or rather a nominal torque of $33 \mathrm{Nm} .{ }^{1}$ In overboost operation, the motor is even capable to generate more than $100 \mathrm{Nm}$ of torque. Consequently, four of these engines are strong enough to accelerate the car mass of approximately $600 \mathrm{~kg}$. Of course, the mechanical construction to carry these drives has to be very stiff. To fix the motor, its shaft extension is mounted via clamping set with $35 \mathrm{~mm}$ bore which guarantees sufficient reserves in torque transmission and holding force.

As importance is attached to $\mathrm{X}$-by-wire technique and fault tolerant software in the future, there are no mechanical brakes. So deceleration is managed just by the motors, which act as generators, whereas part of the energy is fed back. To prove really robust control, there is no fallback solution with mechanical brakes in case of emergency. Brake torque can be adjusted within the motor controller, regarding the limit for recuperation which the batteries can bear up. For safety reasons, up to now the maximum speed of $15 \mathrm{~m} / \mathrm{s}$ hasn't been reached, test runs were accomplished at velocities below $5 \mathrm{~m} / \mathrm{s}$ with a maximum brake torque current of $40 \mathrm{~A}$ for each wheel.

\footnotetext{
${ }^{1}$ values for one traction drive module
} 
Inside the motor, there is a little rotary encoder, generating position data and thus offering the prerequisites for precise velocity control and synchronized motion control of the four in-wheel motors. For a first prototypic implementation, the original encoders with 8-bit resolution are retained (type AM256, RLS). In addition, there is also a silicon temperature sensor (type P/N KTY 84-150, Philips), monitoring the thermal of the motor winding.

Chassis suspension was developed due to the demands of the car, namely the option to fully rotate the driving wheel (vertical axis through the middle of the wheel). The result of these premises are two linear rail sleeve bearings, equipped with two standard bead-spring units. These units are derivated from scooter chassis, arranged in pairs in order to result in more payload and higher rigidity.

\section{Steering Drive Unit}

To have the option to completely revolve each of the car's wheel drives, specially constructed carriage is used. To reach large steer angles, conventional steering linkages couldn't be utilized, but an alternative design was necessary. The chassis, that comprises the wheel hub motor, the tire and the shock absorber, is mounted to the car's main frame by means of a plate, which is rotationally supported. In order to save constructed space, a single heavy-duty crossed roller bearing is used to pivot the whole driving unit against the car's frame. Steering actuation is induced by an energy-efficient brushless DC motor with integrated and space-saving vector control unit, coupled to a worm gear (gear ratio: 50:1). This assembly allows powerful movement at maximum speed of $600 \% \mathrm{~s}$, and guarantees directional stability in case of a blackout due to self-locking of the worm gear. In order to detect the precise angle of rotation of the wheel, an incremental 12-bit encoder is attached to the motor shaft, which means a theoretical resolution below $0.002^{\circ}$ (gear ratio included). Due to backlash and transmission errors of the worm gear, the real mechanical resolution is about $0.5^{\circ}$. To reference dedicated home and extreme positions, the system includes three hall sensors together with three solenoids, which are mounted on the parts of the chassis that are moving against each other. The overall steer angle is mechanically unlimited, but restricted to almost $360^{\circ}$ because of the cables.

In the explosion view of Fig. 3, both traction and steering drive unit are depicted. Additionally, the assembly of the steering and traction drive module with corresponding axes is shown in Fig. 4. For detailed descriptions of alternative steer and drive units, we refer the reader to Goris [11].

\section{Kinematics}

The kinematics of the presented car is accompanied by a non-holonomic vehicle, in which the turning mechanism has to be precisely controlled.

There are six distinct driving modes corresponding to the various needs (cf. Fig. 5). The implementation of these modes is supported by a so called best performance curve feature of the motor controllers ACD 4805 [12].

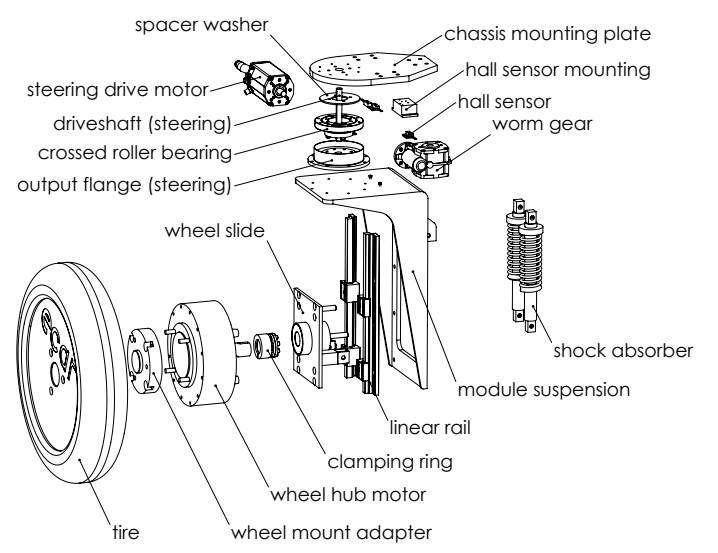

Fig. 3. exploded view of the drive module

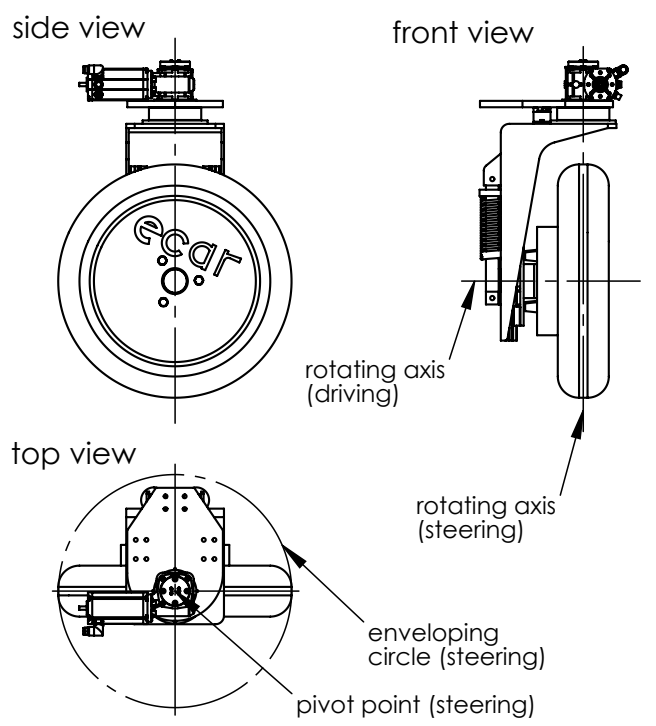

Fig. 4. module assembly with marked axes

In the default mode, steer angles of front axis and rear axis are controlled according to Ackermann angle [13], [14].

Whereas the above mode is realized at higher velocities, driving in strongly related slow mode is characterized by a steering of the two different axes being controlled vice versa (positive and negative angles). At this, the proportion of the angle of the rear axis and the angle of the front axis can be predetermined arbitrarily such that the car is driving around a common pivot point. This mode decreases turn radius on the one hand and improves maneuverability at low speed on the other hand.

The parallel mode is used when all the wheels should be oriented to the same direction, which allows laterally movement into parking lots or lane change.

As soon as all the wheels are turned in a way that they are arranged along a common circle, thus being able to rotate the car around the center of the circle, the rotational mode is activated. 


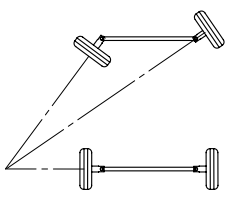

(a) default mode

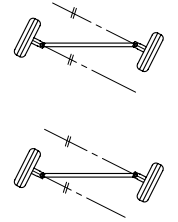

(c) parallel mode

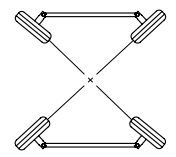

(e) parking mode

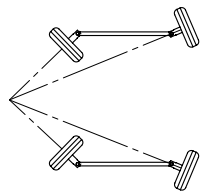

(b) slow mode

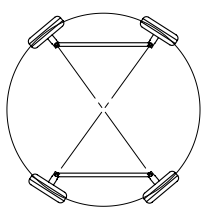

(d) rotational mode
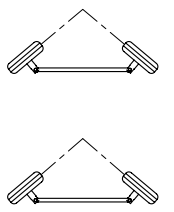

(f) emergency mode
Fig. 5. driving modes

The parking brake mode is performed when all the wheels are arranged like a cross to prevent the car from inadvertent rolling because of the lack of stopping brakes.

During the emergency brake mode the wheels are pairwisely slightly oriented like a V-shape, similar to a plough, in order to be able to stop in the case of failure of the engine brake due to the non-existence of any mechanical brakes (cf. disproportional depiction in Fig. 5f). As a matter of course, this mode has to be carefully parameterized before execution, otherwise the car could get out of control.

\section{HARDWARE AND SOFTWARE OUTLINE}

In the following, insight is given into issues in terms of hardware and software related aspects. The hardware design consists of a distributed component based control concept (cf. Fig. 6). Three microcontroller-boards and one computer for HMI purpose are used to build the basic architecture. Each axis has one microcontroller-board, sending control data for steering drive and traction drive, receiving superordinate commands from an additional third main microcontrollerboard, all within a single Ethernet network. The last one also serves as interface to the HMI computer, linked via UART (Universal Asynchronous Receiver Transmitter). Furthermore, each drive (steering drive, traction drive) has its own motor amplifier or rather controller, which is conducted by means of CAN (Controller Area Network) or rather CANopen.

The HMI computer is based on an Intel Atom dual core processor platform with an ITX mainboard including graphics chipset. This controller receives haptic input from the joystick and the touchscreen monitor, sends control signals to the inferior microcontroller-boards, and also allows to

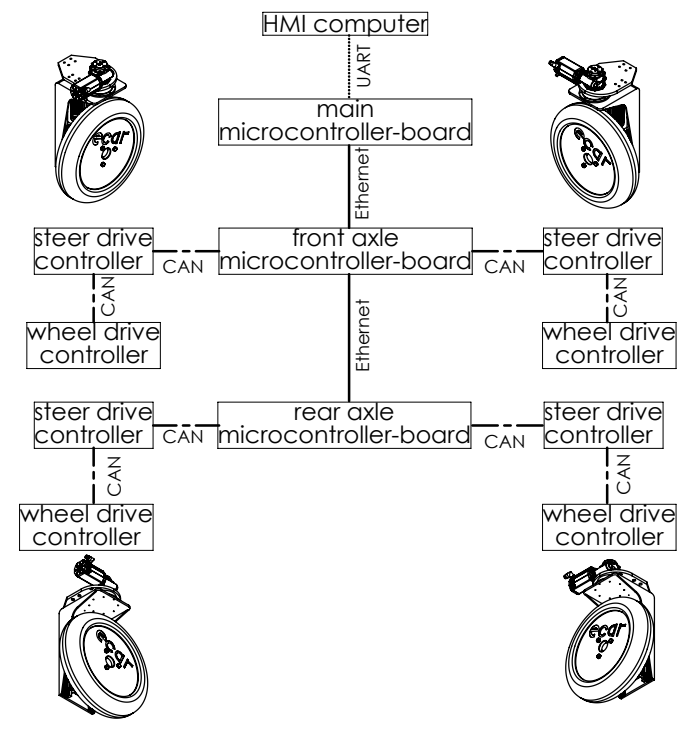

Fig. 6. hardware architecture

give visual feedback to the driver by means of the display. The main task of the HMI is to transmit superordinated control signals to the four steering und traction drive units via communication with the inferior microcontroller-boards using a special Ethernet bus.

Two microcontroller-boards (Luminary Micro Stellaris LM3S8962 with an ARM Cortex M3 32-bit microcontroller), one for the management of each of the two axes, offer a new Ethernet based interface in compliance with IEEE 1588 (precision time protocol [15]) as well as CAN interface. Through the CAN controllers, communication is feasible with the motor controllers on the one hand and with the superior main microcontroller-board via embedded Ethernet controller on the other hand. Interoperability to aforementioned IEEE 1588 offers real-time communication with synchronization of several clocks, comparable to FlexRay [16], [17]. Thus the clocks of different devices such as motor controllers for steering and driving can be handled with adequate sensitivity.

Here the wheel motor controllers ACD 4805, offering flux-vector control techniques, are used in speed control mode (torque control mode would also be feasible) [12]. A closed loop speed regulation within four-quadrant operation is employed, where speed set points (command speed) are accepted as input and torque commands (torque current) are produced as output. The integrated current controller (so called vector control) controls the torque producing current, that is to say it computes the adequate motor voltage being realized by pulse width modulation.

The network communication is realized via Flexible TimeTriggered Ethernet (FTTE), a specially implemented realtime Ethernet protocol with integrated precision time protocol (PTP) according to IEEE 1588 standard. PTP guarantees precise synchronization of clocks, which is necessary because of many time-triggered network communication messages that have to be transmitted. 
For a first step, FreeRTOS was chosen as operating system. This decision was motivated by cost-effectiveness due to open source, portability to a lot of different platforms, advantages in the domain of time synchronization ${ }^{2}$ and last but not least the potential to get the written code certified according to IEC 61508 for SIL3. As the above named operating system is a strongly related product of SafeRTOS, later on reusability for implementation within SafeRTOS is practible without effort.

\section{PREVIOUS RESULTS}

As to be seen in the previous graphics in Fig. 5, our electric car can fulfill extremely flexible movements due to the fourwheel steering. Tests in a private basement garage could prove this motion capability. Because of easy to use HMI devices, different drivers, also ones not being involved in the project, were able to use the car without long time for familiarization. For our first tests, we set a speed limit up to about $5 \mathrm{~m} / \mathrm{s}$ for safety reasons. At the time of the tests no brake resistors were included, thus braking energy could just be absorbed by charging the batteries.

To prevent overloading of the wires and batteries, presently a limit of $40 \mathrm{~A}$ for each drive module is preset to restrict the current that can be fed back to the energy system. This limit is insufficient in case of emergency, thus the above named emergency mode must back up the generator brakes. Accordant braking tests remain to be conducted.

For cost requirements, a first integration of three hall sensors for detection of limit stop of each steering drive (2 sensors) respectively a basic reference position (1 sensor) was used, which turned out to be inappropriate. Each time after a re-start recalibration was necessary to be able to steer the car properly later on. Consequently, a new absolute encoder will be fixed to the driven shaft of the steering drive unit, avoiding problems with the need for a startup process respectively finding a reference position at the beginning. Due to backlash of the worm gears of the steering unit, a mechanical precision of approximately $1^{\circ}$ is the limit currently, which exceeds the precision of the encoder resolution.

Beyond, also the strong relation between steering motor current and velocity is noticeable. In a stationary condition, the motors have to generate a maximum of torque induced by high current (up to $50 \mathrm{~A}$ ), because of static friction of the material combination of the tire (rubber) and the floor (e.g. asphalt). Up to now a maximum torque of about $85 \mathrm{Nm}$ was measured for steering a drive unit of the rear axle, whereas the unit at the front axle gets by on about $50 \mathrm{Nm}$, which is due to the different wheel loads according to the car's center of gravity. Once the car is moving, this current respectively torque dramatically decreases to values of less than $30 \%$.

Moreover, motion drive motor controllers have to be exactly parameterized and the clocks of the microcontrollerboards must be precisely synchronized to result in a wellbalanced motion of the whole car. In accordance with several

\footnotetext{
${ }^{2}$ while periodically accessing tasks, simultaneous use of other timers is assisted
}

measurements, the inaccuracy of already adjusted clocks of the axle controller-boards is in the range of $9.5 \mu \mathrm{s}$ at present. Imprecision in parameters of either wheel or steer drives result in slight differences in rotary speed, thus marginal jerking of the wheels is perceptible on the seat of the car. Currently the straight-ahead travel is mainly downgraded due to the relatively high backlash of the gearboxes of the steer drive units. If the worst comes to the worst, the angle errors of the front axle and the rear axle perform inversely, hence a maximum angle of deviation of the whole car results (unconsidered taking corrective action by means of the joystick). Based on the geometry of our car, Fig. 7 shows the correlation between the angle errors caused by backlash of the gearboxes of the steer drives and the deviation of linear movement or rather unintentional curve radius. Various angles are depicted. Values highlighted in red mark the aforementioned worst case for our current setup.

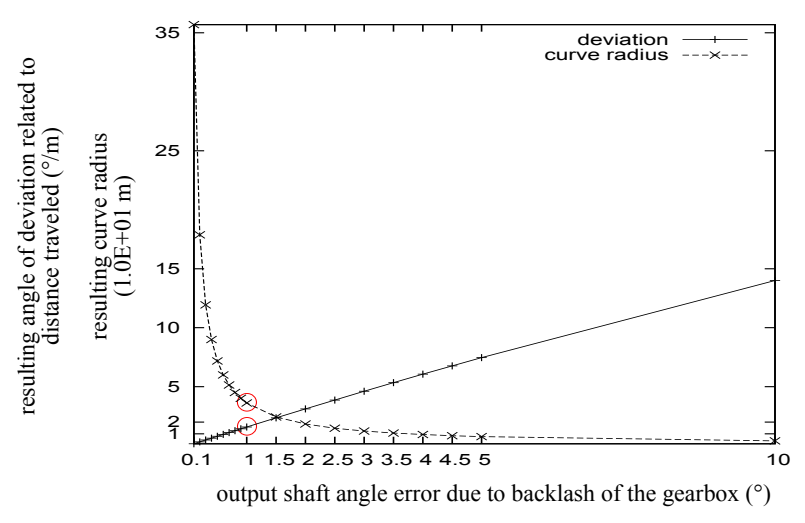

Fig. 7. deviation of straight-ahead travel

Due to relatively lightweight wheel hub motors and small motorcycle tire width, the unsprung weight of each drive unit (less than $25 \mathrm{~kg}$ ) is not too high compared to the sprung mass of the car (front axle $250 \mathrm{~kg}$, rear axle $350 \mathrm{~kg}$ ) and thus performance deficiency in terms of driving comfort is of no significant consequence at the prevalent velocities.

To get an impression of the above specified electric X-bywire car, the prototypic setup of the whole car is presented in Fig. 8, showing the digital mock up and the real car during an exhibition statical with turned in front wheels.

\section{CONCLUSIONS AND FUTURE WORKS}

In the sections above, a new alternative electric car concept was introduced, pointing out the basics of this development. The main idea was to build up a new concept car, by means of which latest innovations can be flexibly implemented and tested. Thus, this car is not meant to be a ready-to-use vehicle for broad mass-production, but to give impulses for new options of electric drive units, and control based on software against the background of fault tolerance and security.

As the project affects various fields of development, further advancement is possible in each of the classical domains of mechatronics, such as mechanics, electronics and software. For there is focus on sophisticated software 


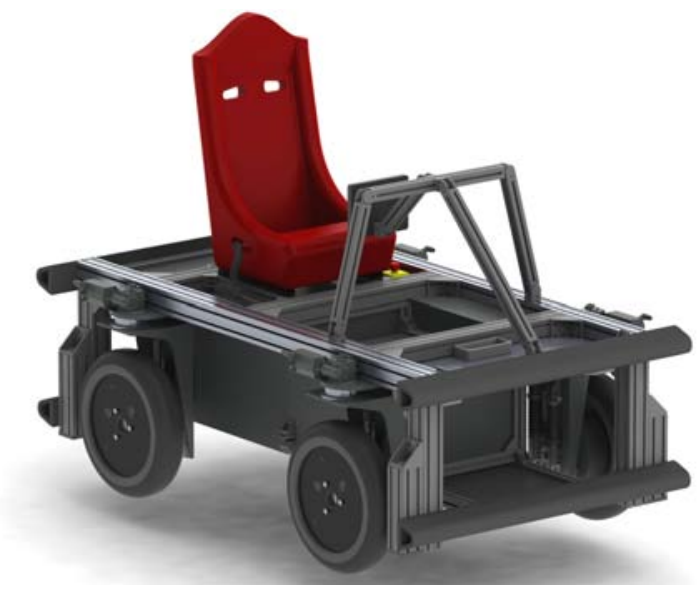

(a) digital mock up

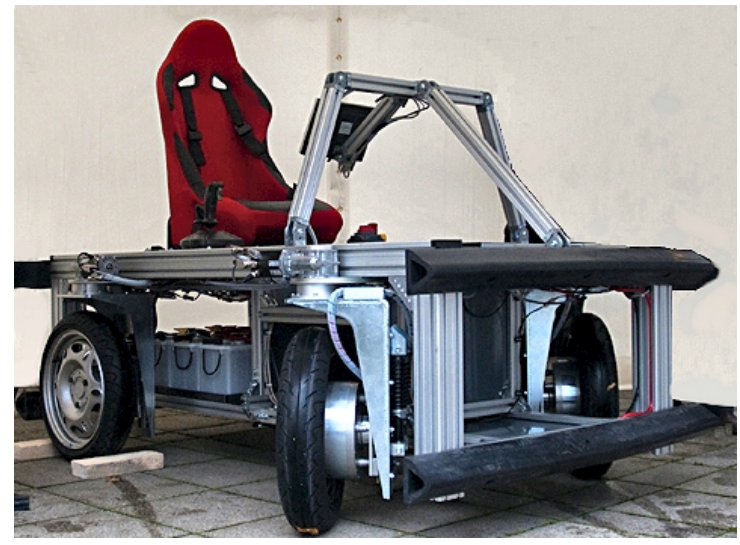

(b) real setup

Fig. 8. pictures of the electric car

development later on, mechanics and electronics just have the purpose to form the physical basis.

The mechanics will be improved by means of steering drive gearboxes with less backlash and the electronic concept will be upgraded to lithium-ion batteries and real recuperation technology through the integration of battery management and brake resistors for the case of emergency. Probably several more devices such as laser range finder or infrared sensors will be added to enhance the sensor qualities of the car. Haptic feedback HMI is not implemented yet, but intended to be included in the future. Therefore, a new force feedback joystick will be used.

In the range of software, the already conceived architecture will be carried on, especially in terms of safety. Here a lot of work will be done to make the car safer respectively fail-safe, on the one hand via advanced software modules, on the other hand by means of redundant devices, e.g. controllers or even cables. Of course, more and more physical models have to be integrated within the car's control, such as four-wheel torque control to stabilize the system at higher velocities or in curves, for instance similar to one of the approaches of accordant experts in this field [18], [19], [20].

Moreover, a new special operating system for electric cars with the aim of real-time Ethernet communication and modular redundancy management is planned to be designed. The modules will be divided into interfaces for the driver and the drivetrain respectively the chassis. Main goal again is the distributed, component based control with FTTE.

\section{ACKNOWLEDGMENTS}

The authors gratefully acknowledge the contribution of fortiss $\mathrm{GmbH}$, SMB Maschinenbau $\mathrm{GmbH}$, Perm Motor $\mathrm{GmbH}$ and Dunkermotoren $\mathrm{GmbH}$.

\section{REFERENCES}

[1] J. Voelcker, "Top 10 tech cars," IEEE Spectrum, vol. 44, no. 4, pp. 30-31, April 2007.

[2] C. Chan, A. Bouscayrol, and K. Chen, "Electric, hybrid and fuel cell vehicles: Architectures and modeling," IEEE Transactions on Vehicular Technology, 2009.

[3] B. Kattentidt, "Intelligent electromechanical drive systems for motor cars," in Proceedings of the 24th Annual Conference of the IEEE, vol. 4, 1998, pp. 2295-2300.

[4] (2010, January) power technology. A123SYSTEMS, Inc. [Online]. Available: http://www.a123systems.com/a123/technology/power

[5] (2010, January) the solution. better place. [Online]. Available: http://www.betterplace.com/solution/

[6] T.-C. Lee and S.-C. Wong, "Promoting the wider use of electric vehicles in hong kong: A strategic proposal," in International Conference on Power Electronics Systems and Applications, Hong Kong, 2009, pp. $1-8$.

[7] F. Zhang and P. Cooke, "The green vehicle trend: Electric, plug-in hybrid or hydrogen fuel cell?" Centre for Advanced Studies, Cardiff University, Tech. Rep., 2009.

[8] C. Chan, A. Bouscayrol, and K. Chen, "State of the art of electric, hybrid and fuel cell vehicles," in Proceedings of the IEEE, vol. 95, no. 4, April 2007, pp. 704-718.

[9] (2010, January) concept car pvio 2. Nissan K.K. [Online]. Available: http://www.nissan-global.com/EN/PIVO2/

[10] J. Hirscha, D. Bassan, C. Lahaye, and M. Goede, "Aluminium in innovative light-weight car design," in International Conference of Innovative Developments for Lightweight Vehicle Structures, Wolfsburg, Germany, May 2009, pp. 101-114.

[11] K. Goris, "Autonomous mobile robot mechanical design," Ph.D. dissertation, Vrije Universiteit Brussel, 2005.

[12] ACD 4805 Drive Motor controller for brushless permanentmagnet motor - user manual, 1st ed., PERM Motor GmbH, Breisach, Germany, 2009.

[13] M. W. Choi, J. Park, B. Lee, and M. Lee, "The performance of independent wheels steering vehicle (4ws) applied ackerman geometry," in International Conference on Control, Automation and Systems, Seoul, Korea, October 2008, pp. 197-202.

[14] K. Yao, Y. Wang, Z. Hou, and X. Zhao, "Optimum design and calculation of ackermann steering trapezium," in Proceedings of the 2008 International Conference on Intelligent Computation Technology and Automation, vol. 01, 2008, pp. 1248-1252.

[15] Precision Clock Synchronization Protocol for Networked Measurement and Control Systems - International Standard IEEE 1588, 2nd ed., IEC/IEEE, February 2009.

[16] J. Duan, L. Zhu, and Y. Yu, "Research on flexray communication of steering-by-wire system," in IEEE Intelligent Vehicles Symposium, June 2009, pp. 824-828.

[17] Y.-N. Xu, Y. Kim, K. Cho, J. Chung, and M. Lim, "Implementation of flexray communication controller protocol with application to a robot system," in 15th IEEE International Conference on Electronics, Circuits and Systems, 2008, pp. 994 - 997.

[18] F. Tahami, R. Kazemi, and S. Farhanghi, "A novel driver assist stability system for all-wheel-drive electric vehicles," IEEE Transactions on Vehicular Technology, vol. 52, no. 3, pp. 683-692, May 2003.

[19] S. Sakai, H. Sado, and Y. Hori, "Motion control in an electric vehicle with four independently driven in-wheel motors," IEEE/ASME Transactions on Mechatronics, Vol. 4, No. 1, 1999, vol. 4, no. 1, pp. 9-16, 1999.

[20] L. Feiqiang, W. Jun, and L. Zhaodu, "Motor torque based vehicle stability control for four-wheel-drive electric vehicle," in Vehicle Power and Propulsion Conference, 2009, pp. 1596-1601. 